\title{
Improved Butterfly Optimization Algorithm: PI Controller for 7-Level Inverter
}

\author{
Meghna Sangtani \\ University of Michigan, Ann Arbor, Dearborn, United States \\ meghnasangtani12@gmail.com
}

\begin{abstract}
In environmental parameters, the Photovoltaic (PV) system generates electricity which varies with deviations as well as at the Maximum Power Point (MPP) the PV network will operate. In addition, to improve the effectuality and also the Renewable Energy Sources (RES) performance, the energy storage devices can act as a possible solution. Moreover, this work tries to set up a control model using an optimization algorithm aided PI controller for the seven-level inverter. On the basis of this, the Proportional Integral (PI) controller increases are attuned dynamically using the Improved Butterfly Optimization Algorithm (IBOA). The increase must be maintained so that the error between the fault signal and reference signal must be the least. Therefore, using the developed optimized PI controller, superior dynamic performance can be attained. At last, the developed model performance is evaluated with the conventional models regarding various metrics, and the proposed method shown its dominance over the conventional models.
\end{abstract}

Keywords: Electricity, MPP RES, Optimization Algorithm, PV, PIController.

\begin{tabular}{ll} 
Nomenclature & \\
\hline Abbreviations & Descriptions \\
\hline THD & Total Harmonic Distortion \\
DGs & Distributed Generations \\
PV & Photovoltaic \\
FC & Fuel Cell \\
ERWCA & Evaporation Rate Based Water Cycle Algorithm \\
MG & Microgrid \\
PCC & Point of Common Coupling \\
CDMs & Clean Development Mechanisms \\
2DOF-PI & 2-degree of freedom PI \\
NN & Neural Network \\
MFO-PI & Modified Fractional Order PI \\
ARPI & Adaptive Reference PI \\
VSI & Voltage Source Inverters \\
IMC & Internal Model Control \\
LVRT & Low Voltage Ride through \\
DG & Distributed Generation \\
IPDT & Integrating Plus Dead Time \\
BESS & Battery Energy Storage System \\
SOFC & Solid Oxide Fuel Cell \\
EHA & Elephant Herding Algorithm \\
P\&O & Perturbs and Observe \\
PI & Proportional Integral \\
MPPT & Maximum Power Point Tracking \\
SRG & Switched Reluctance Generator \\
POC & Point of Connection \\
VSC & Voltage Source Converter \\
PCC & Point of Common Coupling \\
PCU & Power Conditioning Unit \\
\hline
\end{tabular}




\section{Introduction}

The electrical energy demand is rising exponentially worldwide. To convene the energy requirements it is usual to exploit power from centralized utilities that tend to maximum distribution and maximum transmission losses. In addition, aforesaid utilities are mainly leave carbon footprint as well as fossil fuels. Conversely, ties together renewable energy-based power produces like minimum emission of carbon as well as economically feasible. To increase the transmission networks, it is not always possible to fulfill the increasing energy demand. Hence, producing power close to the customer location can advantage the power utilities regarding the loss of power as well as enhanced system reliability. DGs are decentralized power generation sources that are compressed, competent, and mainly based upon renewable energy like PV system, FC, and wind energy, so on. Hence, renewable energy idea-based MG and its management has been developed as important fields of research in the energy segment [1].

Numerous confronts are related to linking PV systems into electrical networks. From aforesaid confronts the deviation of produced PV power because of environmental alterations, non-linearity in the characteristics of PV, non-linearities in power electronics modules, reliability as well as stability. At the time of abnormal operating circumstances such as grid faults another technical confronts are exhibited. Aforesaid faults of the grid are not restricted to short circuits at PCC among PV systems as well as grid, and sagging circumstances related to abruptrise in load. These faults considerably ascertain the condition of PV systems if linked or unlinked to the grid. Few severe basics were proposed to ascertain the link or unlink of PV systems from the grid. In a plot of PCC voltage vs. time (grid code) aforesaid basics were indicated [2].

The MPPT controller appears to be an integral module of PV systems and these methods attain complete power from a PV array in several environmental circumstances. Naturally, these services might be recycled and refilled. Hence these resources may be presumed as infinite for specific practical reasons, apart from conventional waning fossil fuels. With the advancement and expansion of green and sustainable energy sources the global energy disaster has reinvigorated. All over the world Organizations are applied CDMs. To increase the solar panel's competence, the MPPT technique is required. There were several MPPT technologies, such asFuzzy Control, P\&O, Fractional Short Current, NN Control, Incremental conductance, Fractional Open Circuit Voltage, so on. Due to their uncomplicated functioning and several other economic purposes, the $\mathrm{P} \& \mathrm{O}$ and Incremental conductance were generally exploited. Hence, MPPT is significant for PV power generation systems to work at the utmost point. Nevertheless, the MPP also alters with the irradiation level and temperature because of the nonlinear PV module characteristics [3].

The main purpose of this paper is to propose an optimized PI controller, whereas the control increases are fine-tuned using an optimization algorithm. Therefore, a novel Improved Butterfly Optimization Algorithm (IBOA) is proposed in this paper. Finally, the performance analysis is performed by comparing the proposed method with the conventional models.

\section{Literature Review}

In 2020, D Bensiker Raja Singh and, R Suja Mani Malar[1], focused by means of the well-organized control model. The contribution of the developed model was to discover the effectual model to maintain a dynamic and steady-state analysis. It was used to ascertain the machine performance and the parameters. To increase the source voltage and AC to DC transformation was carried out using the Quasi Z- source network. At a standard speed, to drive the induction motor, few controllers were analogized as well as a fraction PI controller was performed and it was selected at a top-notch level.

In 2020, Velamuri Suresh et al [2], proposed a decentralized control scheme on the basis of the MFOPI and 2DOF-PI controllers for the competent operation of an independent MG. The independent MG comprises SOFC and PV system as DG,BESS as a storage unit as well as several AC and DC loads. To control VSI the MFO-PI controller was used, to control various DGs and BESS 2DOF-PI was exploited. To optimally tune the developed controllers an ERWCA was used.

In 2020, Ayman Alhejji and Mohamed I. Mosaad [3], worked on the improved combination of PV models into electrical networks controlling both inverters as well as a converter. For the inverter, an ARPI controller was developed to enhance system performance via aiding LVRT ability, as well as smoothing PV-produced power fluctuations at the time of variable environmental circumstances.

In 2020, Ujjwal Manikya Nath et al [4], developed a PI controller on the basis of the IMC model. Proportional and integral (kI) increase of developed IMC-PI controller for IPDT procedure was computed on the basis of the increase and phase margin. For the IMC-PI controller, the appropriate option for the single tuning parameters that were closed loop time constant plays an important role to attain the preferred closed-loop response. 
In 2020, Mohamed F. El-Naggar et al [5], developed an optimal PI controller optimized using EHA to control grid-tied 4-phase 8/6 SRG. The LVRT ability of the grid-tied SRG aforesaid controller was developed during faulty circumstances which affect SRG output voltage using an adaptable off-delay angle of the generator. At the POC of the SRG, these faulty circumstances would take place to the wind speed variations.

\section{System Modeling}

Fig. 1 demonstrates the DG combined into a 4 wire distribution network and 3 phase by exploiting a seven-level inverter. This paper uses 3-phase, 3-leg, 7-level VSC. Using a PCU the RES-adjusted DG unit is connected to dc-link and they are decoupled from its usefulness grid using a dc-link capacitor $\left(\mathrm{C}_{\mathrm{dcl}}\right.$ and $\mathrm{C}_{\mathrm{dc} 2}$ ) and it permits automatic control of the converter on both sides of dc-link [6]. To the power grid, the seven-level inverter output is linked through an LCL filter. In a series manner, the damping resistor $R_{d}$ has been linked to the filter capacitor $\mathrm{C}_{\mathrm{f}}$ and it presents passive damping. Additionally, a wide range of balanced as well as unbalanced local non-linear/linear loads was linked to the PCC.

A boost converter (or any dc-dc converter) in MPPT links PV array with the load. MPPT technique enhances duty ratio (of this converter) so that PV array is worked at a voltage (or current) equivalent to utmost powerpoint". A solar power system or PV system is a power system modeled to provide functional solar power via photovoltaics". To convert DC into AC a VSC is exploited. It is a device and converter here, the dc voltage posses' one polarity, as well as reversal of power, occurred using the current polarity reversal.

Using cascading 3 full-bridge inverter circuits the seven-level inverter is attained. In series, 3 fullbridge inverters are linked as well as a single-phase output is used. From a separate DC source, every full-bridge is subjected. VDC indicates volts of direct current. It needs power supplies of sensitive electronics to encompass a correcting circuit that converts the irregular current to direct current. The voltage and polarity of irregular current receive the form of a sine wave".

Generally, a usefulness grid is for-profit electric power distribution system which uses electricity from a generator for instance fossil fuel generator and boiler, water turbine, diesel generator, wind turbines, and so on, transfers it against a definite distance, and subsequently uses electricity down to customer during a distribution".

\section{Proposed Optimized PI controller for control system}

The proposed PI controller is used for the q0-axis control of the seven-level inverter and it is shown in Fig 2. The PI controller inputs are voltage produced by reducing error among the fault signal as well as reference signal [7]. The increase of the PI controller was produced arbitrarily with reference to these input waveforms.

The PI controller increases must be fine-tuned so that error among the fault signal as well as the reference signal should be minimized. Here, the proposed IBOA is used to fine-tune the PI controller gains.

\subsection{Objective model}

The proposed model aspires to reduce the error among the fault signal and reference signal. In addition, the total harmonic distortion and reactive power possess to be reduced for superior system performance. Here, the integral gain $\left(G_{I}^{\prime}\right)$ and proportional gain $\left(G_{P}^{\prime}\right)$ of the PI controller which is subjected as input to the solution to encode. Eq. (1) indicates The objective model of the fitness function (OF ) of the developed method, whereas $R_{p}$ signifies the reactive power reduction, E signifies the error among the fault signal and the reference signal, THD signifies the total harmonic distortion, and $\mathrm{P}_{\mathrm{r}}$ signifies the real power maximization. THD is stated in Eq. (2), whereas $V_{2}^{2}+V_{3}^{2}+\ldots . .+V_{n}^{2}$ is the power in the supply because of the harmonics and $\mathrm{V}_{1}^{2}$ the power of the basic supply.

$$
\begin{aligned}
& \text { Fitness Function }=\mathrm{OF}=\operatorname{Min}\left(\mathrm{E}+\mathrm{THD}+\mathrm{R}_{\mathrm{p}}+\frac{1}{\mathrm{P}_{\mathrm{r}}}\right) \\
& \mathrm{THD}=\sqrt{\frac{\mathrm{V}_{2}^{2}+\mathrm{V}_{3}^{2}+\ldots . .+\mathrm{V}_{\mathrm{n}}^{2}}{\mathrm{~V}_{1}^{2}}}
\end{aligned}
$$




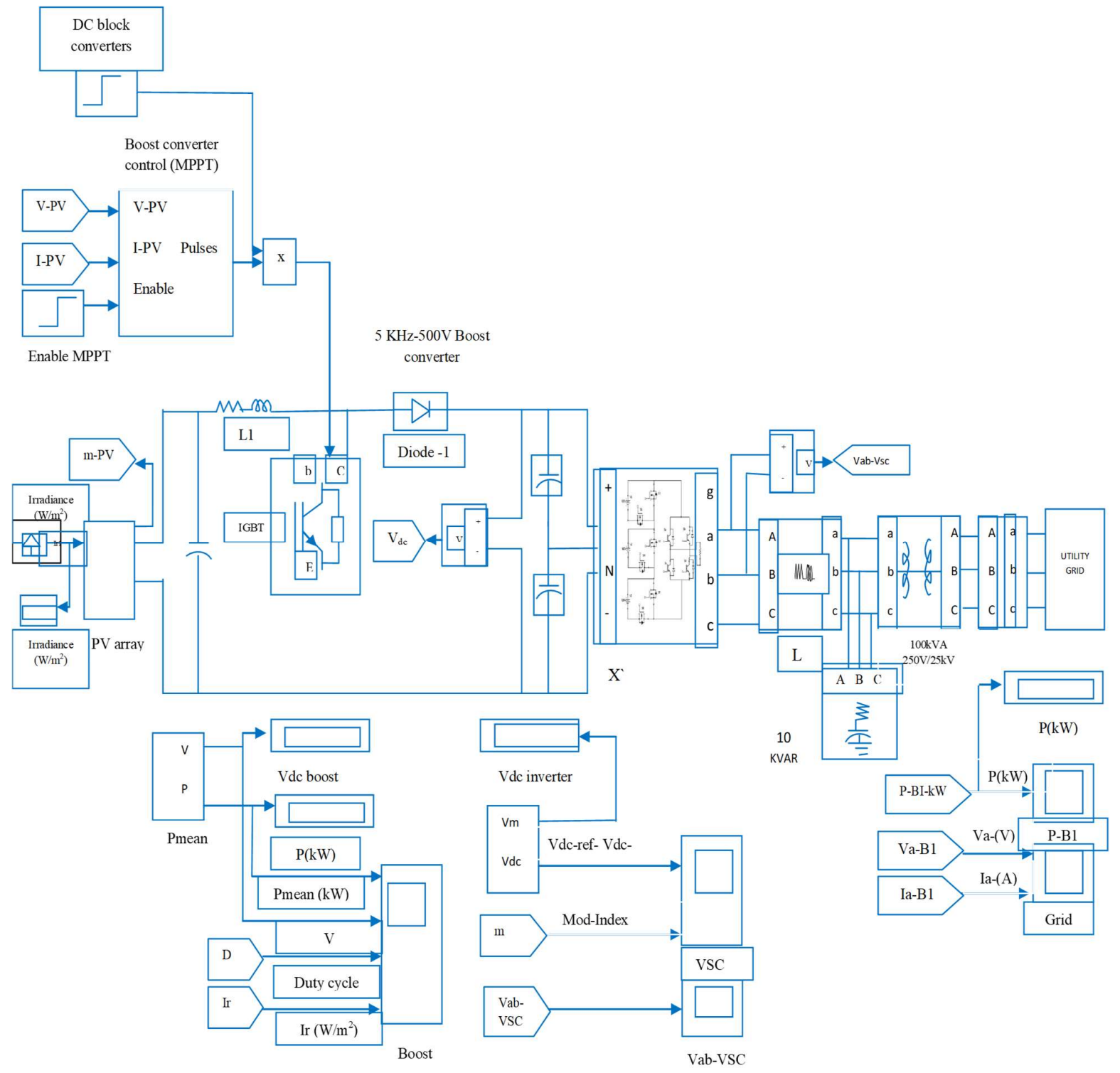

Fig. 1. Block diagram of the proposed model

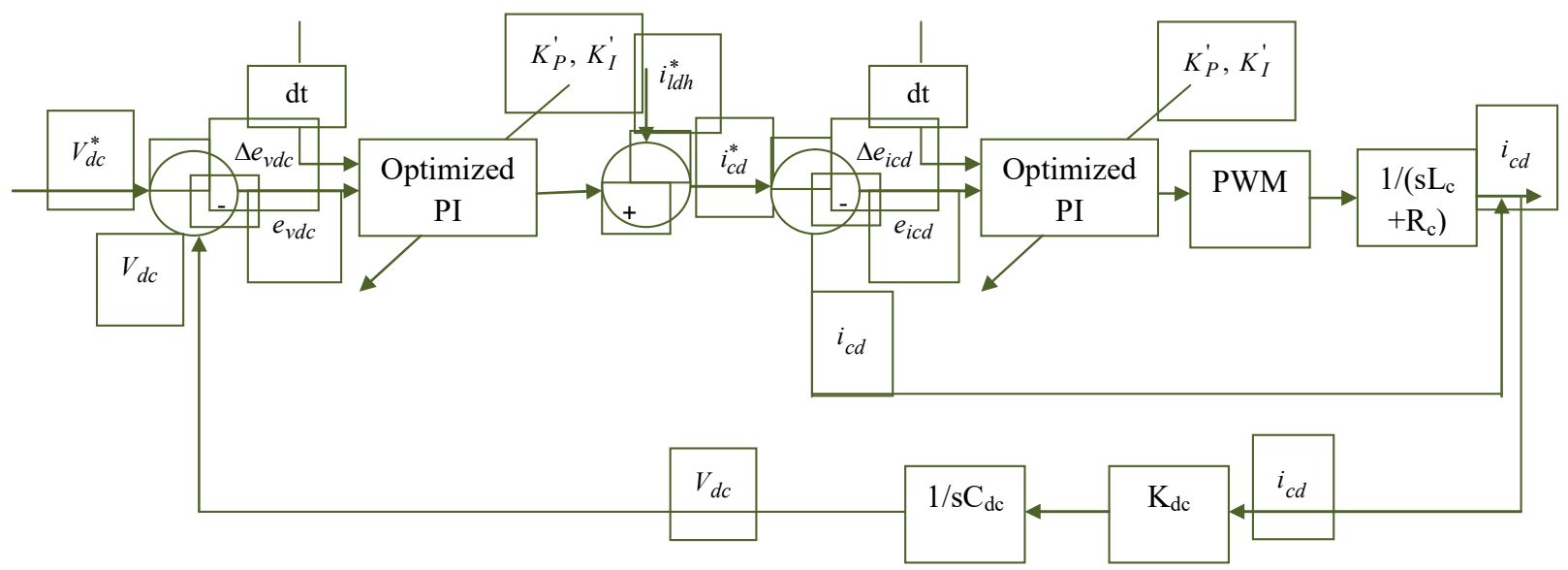

Fig. 2. Diagrammatic representationof the proposed PI controller 


\subsection{Proposed IBOA algorithm}

In [8], BOA is a meta-heuristic technique on basis of the food foraging behavior of the butterflies. The butterflies are the search agents that employ the sense of smell to ascertain the positions of food or mating partner. Each butterfly can produce fragrance with some intensity that is correlated with its fitness. Even though the BOA has exhibited a capable tool to solve several issues, the study on conventional BOA needs more work. Because of the weak exploitation ability of the model, a novel enhanced model, called, the IBOA is proposed to modify the vital parameters of the BOA. In each iteration, the switching probability is the most important decision controller to decide the incidence of either local search or global search. In the conventional BOA, the value of the switching probability is fixed at 0.8 , in that the probability of the exploration phase is superior to the exploitation phase. Thus, the BOA becomes poor in exploitation and might lose the optimum solution because of aggressively exploring the search space. Hence, few modifications are presented, concentrating on an intensive exploitation phase that presents more chances for the solutions to enhance itself.

The fragrance $\mathrm{f}$ is devised as a function of the physical intensity of stimulus as below:

$$
\mathrm{f}=\mathrm{cI}^{\mathrm{a}}
$$

whereas c indicates sensory modality to measure energy form, I indicates the stimulus intensity. I of a butterfly is related to the encoded objective function of the optimization issue. The value of $\mathrm{c}$ is computed as:

$$
\mathrm{c}(\mathrm{t}+1)=\mathrm{c}(\mathrm{t})+0.015 \frac{\mathrm{c}(\mathrm{t})}{\mathrm{MaxI}}
$$

whereas MaxI indicates the utmost count of iterations and $t$ indicates the current iteration number. To the extent that arbitrariness is troubled, in BOA, a user predefined switch probability $\mathrm{p}$, is exploited to switch among these two main stages of attraction as well as arbitrary movement, regarding the global or local search, correspondingly. The switching probability $\mathrm{p}$ is evaluated with the value of $\mathrm{r}$, an arbitrarily produced number in $[0,1]$. If the arbitrary number $r$ is greater than $p$, local search is carried out; else, global search is developed. In global search, a butterfly is capable to sense fragrance from any other butterfly, whereas solutions are updated as below:

$$
x^{t+1}=x^{t}+\left(r^{2} g-x^{t}\right) f, r<p
$$

$\mathrm{g}$ indicates the current optimal solution found between all at the current iteration $t$.

$$
x^{t+1}=x^{t}+\left(r^{2} x_{j}^{t}-x_{k}^{t}\right) f, r>p
$$

$t \mathrm{x}_{\mathrm{j}}^{\mathrm{t}}$ and $\mathrm{x}_{\mathrm{k}}^{\mathrm{t}}$ indicates the $\mathrm{j}^{\text {th }}$ and $\mathrm{k}^{\text {th }}$ butterflies, correspondingly, at iteration $\mathrm{t}$, that is chosen arbitrarily from the solution space. The exponential increase p initiates from 0 and the successive values can be attained by subtracting each value of pp of Eq. (7) from unity. The exponential increment p is used as below:

$$
p p^{t+1}=p p^{t}-e^{\frac{-t}{t+1}} \times \sigma \times p p^{t}
$$

One more alteration is related to Eq. (6) of the conventional BOA. In the local search, the solutions are developed be updated as below:

$$
x^{t+1}=x^{t}+i\left(x_{j}^{t}-x_{k}^{t}\right) f, r>p
$$

This enhancement permits for a larger step where $\mathrm{i}$ indicates a random number among 0 and 1 , and is always greater than the value of $\mathrm{r}^{2}$ in Eq. (6). This aid in evasion from the local minimal and exploring more areas within the search space. In the developed model, the value of sensory modality is computed using the below equation:

$$
c(t+1)=c(t)+0.15 \frac{c(t)}{\operatorname{MaxI}}
$$

\section{Result and Discussion}

The experimentation analysis of the developed model for an optimal PI controller has experimented and the results were shown in this section. Here, the proposed method is compared with the conventional models such as Firefly (FF) [11], Artificial Bee Colony (ABC) [12] and Particle Swarm Optimization (PSO)[10] models. 
In Table 1, the steady-state response analysis for attaining the optimal PI controller by exploiting the proposed technique is shown. Here, the proposed method is $12 \%$ better than the $\mathrm{FF}, 18 \%$ better than the $\mathrm{ABC}$ and $22 \%$ better than the PSO algorithm in terms of rise time.

In Table 2 and 3, the performance analysisof the developed model with the conventional models regarding the THD and fitness function is summarized.For Table 2, the proposed method is 33\%better than the FF, 36\% better than the ABC and $32 \%$ better than the PSO algorithm in terms of best. In Table 3, the proposed method is $26 \%$ better than the $\mathrm{FF}, 22 \%$ better than the $\mathrm{ABC}$ and $28 \%$ better than the PSO algorithm in terms of best .

Table 4 exhibits the analysis of the optimal values of $K_{p}$ and $K_{i}$ increases.

Table 1Steady-state analysis of proposed model

\begin{tabular}{lllll}
\hline Time & FF & ABC & PSO & Proposed model \\
\hline Rise time & 0.000684 & 0.01334 & 0.00873 & 0.00873 \\
Settling time & 0.188 & 0.0187 & 0.01763 & 0.01763 \\
Settling Min & -416.8346 & 331.00043 & 384.11308 & 384.1131 \\
Settling Max & 88.30303 & 331.00043 & 331.00043 & 331.00043 \\
Overshoot & 681.7806 & $7.1008 \times 10-10$ & $7.7603 \times 10-10$ & $7.7304 \times 10-10$ \\
Undershoot & 636.0406 & 0 & 0 & 0 \\
Peak & 416.8346 & 331.00043 & 331.00043 & 331.00043 \\
Peak Time & 0.08 & 0.03 & 0.03 & 0.03 \\
\hline
\end{tabular}

Table 2Performance analysis of proposed model regarding THD

\begin{tabular}{lllll}
\hline Methods & FF & ABC & PSO & Proposed model \\
\hline Best & $3.49033 \times 30^{-08}$ & $3.49033 \times 30^{-08}$ & $3.49033 \times 30^{-08}$ & $3.49033 \times 30^{-08}$ \\
Worst & 3.883335 & 3.30294 & 3.3029 & 3.30293 \\
Mean & 0.37475 & 0.07238 & 0.053 & 0.05047 \\
Median & 0.0822 & 0.00058 & 0.000339 & 0.000339 \\
STD & 0.409928 & 0.224839 & 0.23745 & 0.237373 \\
\hline
\end{tabular}

Table 3Performance analysis of proposed model regarding Fitness function

\begin{tabular}{lllll}
\hline Methods & FF & ABC & PSO & Proposed model \\
\hline Best & 77.7047 & 72.7427 & 76.7226 & 75.3704 \\
Worst & 79.7497 & 74.59262 & 77.3222 & 76.2375 \\
Mean & 79.36245 & 73.407 & 77.70244 & 75.6959 \\
Median & 79.3692 & 73.20726 & 77.73272 & 75.6232 \\
STD & 0.497925 & 0.670705 & 0.550709 & 0.32542 \\
\hline
\end{tabular}

Table 4Performance analysis of optimal values

\begin{tabular}{lllllll} 
Methods & $K_{p 1}$ & $K_{i 1}$ & $K_{p 2}$ & $K_{i 2}$ & $K_{p 3}$ & $K_{i 3}$ \\
\hline FF & 0.7097 & 0.7677 & 0.77777 & 0.44414 & 0.96761 & 0.20415 \\
ABC & 0.52547 & 0.67112 & 0.1995 & 0.26702 & 0.67271 & 0.216 \\
$\begin{array}{l}\text { PSO } \\
\begin{array}{l}\text { Proposed } \\
\text { model }\end{array}\end{array}$ & 0.7097 & 0.7677 & 0.7779 & 0.6276 & 0.0111 & 0.1256 \\
\hline
\end{tabular}

\section{Conclusion}

A seven-level inverter control model was designed using a new optimization algorithm that guarantees the dynamic performance in control generation for PI controller. Therefore, a novel optimization named IBOA was proposed that overcomes the problem in existing methods. Using the fitness function, this proposed model overcomes all the issues and raises the convergence speed. At first, the present analysis was performed subsequently the voltage analysis was examined. Hence, the error between the fault and reference signal was minimized. Here, the steady-state responses namely settling minimum, overshoot etc was performed using the proposed model. At last, in all scenarios, the developed model has revealed the betterment over the conventional models. 


\section{References}

[1] D Bensiker Raja Singhr Suja Mani Malar,"Implementation of Fractional Pi Controller for Optimal Speed Control Of Induction Motor Fed With Quasi Z-Source Converter", Microprocessors And Microsystemsavailable Online 9 October 2020.

[2] Velamuri SureshNikhil PachauriT. Vigneysh,"Decentralized control strategy for fuel cell/PV/BESS based microgrid using modified fractional order PI controller",International Journal of Hydrogen Energy28 November 2020.

[3] Ayman AlhejjiMohamed I. Mosaad,"Performance enhancement of grid-connected PV systems using adaptive reference PI controller",Ain Shams Engineering Journal25 September 2020.

[4] Ujjwal Manikya NathChanchal DeyRajani K. Mudi,"Stabilized IMC-PI controller designing for IPDT processes based on gain and phage margin criteria", IFAC-PapersOnLine12 August 2020.

[5] Mohamed F. El-NaggarMohamed I. MosaadAhmed F. Bendary,"Elephant herding algorithm-based optimal PI controller for LVRT enhancement of wind energy conversion systems". Ain Shams Engineering Journal4 September 2020.

[6] M. Singh, V. Khadkikar, A. Chandra, R.K. Varma, "Grid interconnection ofrenewable energy sources at the distribution level with power-qualityimprovement features”, IEEE Trans. Power Deliv, vol. 26, no. 1, pp. 307-315, 2011.

[7] W.H. Hayt, Engineering Circuit Analysis, Tata Mcgraw Hill Education Pvt. Ltd,2010.

[8] S. Arora, S. Singh, Butterfly optimization algorithm: a novel approach for global optimization, Soft Computing 23 (2019) 715-734.

[9] SK.Mahammad Shareef," Power Quality Enhancement by Optimally Placing the UPQC in the Distribution System: A Hybrid Optimization Model", vol. 4, no. 1, January 2021.

[10] Gayathri Devi K.S,"Hybrid Genetic Algorithm and Particle Swarm Optimization Algorithm for Optimal Power Flow in Power System",Journal of Computational Mechanics, Power System and Control, Volume 2, Issue 2, April 2019.

[11] Chithra.S and R.Madahana Kumari," Economic Emission Dispatch in Renewable Energy Systems using FireFly Algorithm",Journal of Computational Mechanics, Power System and Control,Volume 1, Issue 1, October 2018.

[12] Rashid Khan," Artificial Bee Colony-based General Adversarial Network for Liver Cancer Detection using CT Images",Journal of Computational Mechanics, Power System and Control, Volume 3, Issue 4, October 2020. 